\title{
Closing male-female gaps in clinical care requires addressing entrenched ideas on gender risks
}

- Cite as: CMAJ 2018 October 1;190:E1179-80. doi: 10.1503/cmaj.109-5645

Posted on cmajnews.com on Sept. 13, 2018.

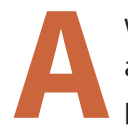

woman having a heart attack has a higher risk of dying than a man, possibly because she won't receive life-saving treatments as quickly. To raise awareness of this gender disparity, the Heart and Stroke Foundation of Canada launched a campaign with a video of a woman sent home from an emergency room in the midst of a myocardial infarction. But eliminating gender gaps in clinical care will take more than a video.

Gender differences in health and disease first started getting substantial attention in the early 1990s, when the US National Institutes of Health (NIH) began requiring federally funded researchers to include women in their research, and the U.S. Food and Drug Administration (FDA) directed companies to enroll female participants in drug studies. Until then, clinical trials for drug approval often excluded women. Health Canada issued its first guidelines mandating the inclusion of women in drug trials in 1997. In 2000, the Canadian Institutes of Health Research (CIHR) established its Institute of Gender and Health to promote research on male-female differences.

Today, the study of gender and sex differences is burgeoning, with clear effects. For example, in 2013, the FDA recommended doctors prescribing one of the most popular sleeping pills (zolpidem, also known by brand names Ambien or Sublinox) give lower doses to women. Health Canada followed with a similar caution in 2014. Studies showed that women's bodies clear the drug more slowly than men's, and that women drive

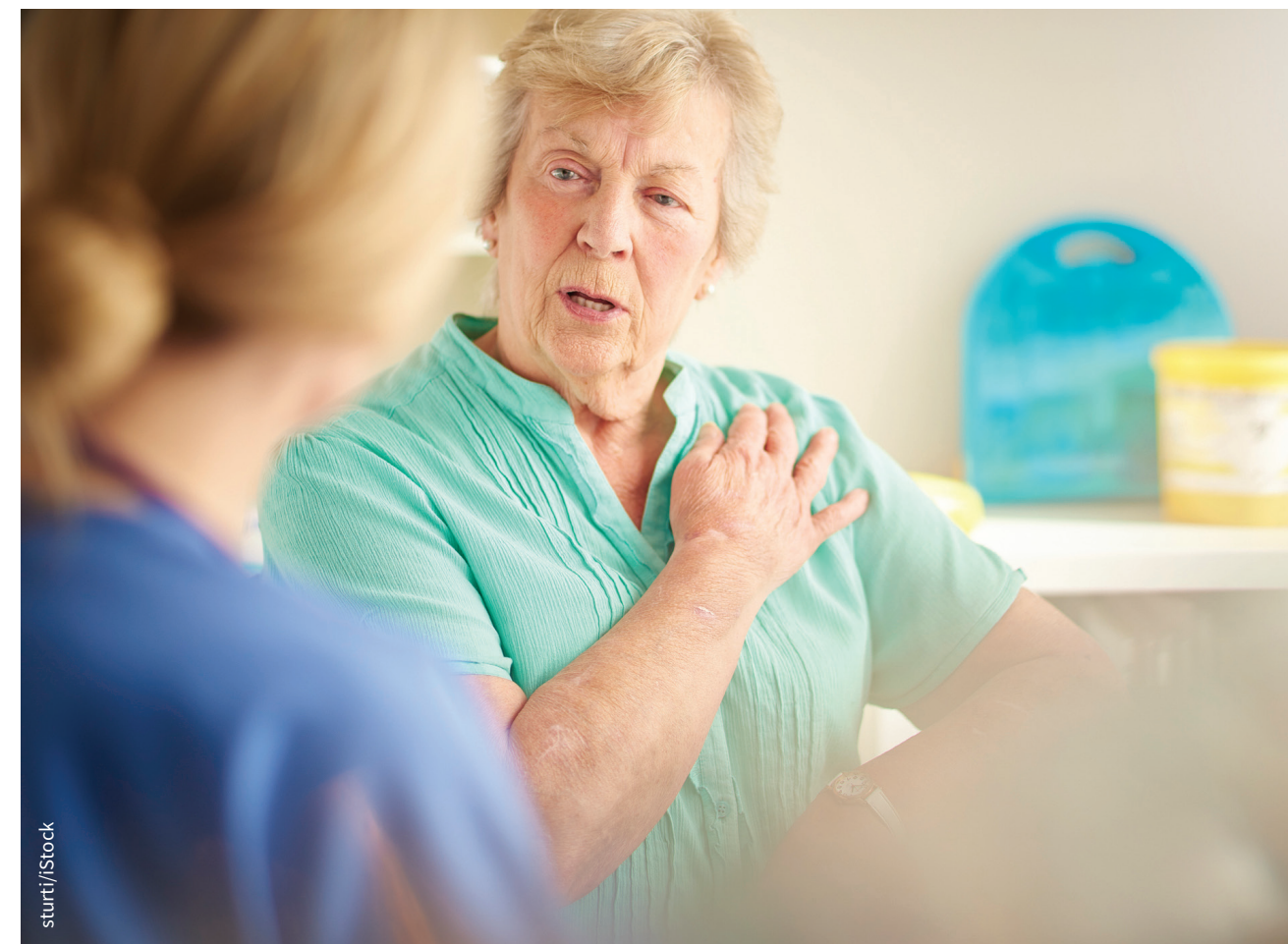

Women are more likely to die from heart failure than men because they aren't admitted for life-saving care as quickly.

worse than men a few hours after taking the pill, putting them at higher risk of crashing.

But in certain areas of medicine, including heart disease, gender differences are entrenched. Dr. Louise Sun and her team at the University of Ottawa Heart Institute have been investigating male-female differences using an Ontario-wide database, and reported this year in CMAJ that women have a higher chance of dying from heart failure than men. She thinks women present with different symptoms from men and may get "written off as having a res- piratory problem," so by the time they finally get treated, they're sicker. Sun and her team also discovered that women with coronary artery disease are referred later than men for bypass surgery, and their outcomes after surgery are worse.

To translate these sorts of findings into clinical care, the CIHR Institute of Gender and Health is promoting what its director, Dr. Cara Tannenbaum, calls "on-the-ground, sustainable change" via designated champions to coach clinicians to incorporate the ideas of sex and gender into medicine. The Canadian 
Cardiovascular Society is working with one such champion, University of Alberta nurse and epidemiologist Colleen Norris, to update its practice guideline on myocardial infarction.

Norris reviewed about 200 randomized controlled trials to form the evidencebase for the new guideline and discovered that most didn't include enough women (women make up only $10 \%$ of participants in some of the trials). Few of the trials stratified by sex to see if the treatment or intervention tested is riskier for women or if outcomes in women were the same as in men. "We can't confirm that these guidelines work for women as well as for men," she said.

Changing clinical practice is also complex because gender bias is unconscious, Tannenbaum said. "It's based on pattern recognition; It's not, 'Oh, she's a woman, I don't want to treat her.'”

The Cleveland Clinic in Ohio is combatting the problem through a systemsbased approach applied equally to male and female patients who present with symptoms of myocardial infarction. In 2014, the clinic implemented a protocol that requires emergency doctors to treat all patients according to a checklist and to send patients for cardiac catheterization without first speaking to cardiologists. A before-and-after study showed the protocol provides female patients with quicker access to life-saving procedures and also appears to narrow the mortality gap between men and women.

Dr. Miriam Shuchman, Toronto, Ont. 